\title{
2pm/V in Poled Bismuth-Zinc-Borate High Index Glass
}

\author{
C. Corbari ${ }^{1}$, L. Chandru' ${ }^{2}$, I.C.S. Carvalho ${ }^{3}$, O. Deparis ${ }^{4}$,F.P. Mezzapesa ${ }^{5}$, P.G. Kazansky ${ }^{1}$, K. Sakaguchi ${ }^{6}$ \\ ${ }^{1}$ Optoelectronics Research Centre, University of Southampton, SO17 1BJ, United Kingdom \\ ${ }^{2}$ Politecnico di Torino, Dipartimento di Scienza dei Materiali ed Ingegneria Chimica, C.so Duca degli Abruzzi 29, 10129 Torino, Italy \\ ${ }^{3}$ Departamento de Física, Pontifícia Universidade Católica do Rio de Janeiro, Rua Marquês de São Vicente 225, Gávea, 22452-900 Rio de \\ Janeiro, RJ, Brazil \\ ${ }^{4}$ Laboratoire de Physique du Solide-Facultés universitaires Notre-Dame de la Paix; rue de Bruxelles, 61; B-5000 Namur, Belgium \\ ${ }^{5}$ National Nanotechnology Laboratory of INFM-CNR,c/o Distretto Tecnologico, Università del Salento, via Arnesano, I-73100 Lecce (Italy) \\ ${ }^{6}$ Technical Research Laboratory, Nippon Sheet Glass Co. Ltd., Itami, Hyogo 664-8520, Japan
}

Thermal poling freezes an intense static electric field $\left(\mathrm{E}_{\mathrm{dc}}\right)$ in glass resulting in a permanent second-order nonlinearity via a rectification process: $\chi^{(2)}=3 \chi^{(3)} \mathrm{E}_{\mathrm{dc}}$. The low third-order nonlinearity limits the $\chi^{(2)}$ of silica glass to $0.7 \mathrm{pm} / \mathrm{V}$. Hence, poling of high $\chi^{(3)}$ glasses offers an attractive route towards glass based integrated electro-optical modulators and frequency converters for which $\chi^{(2)}$ of the order of $5 \mathrm{pm} / \mathrm{V}$ or higher would be desirable. In the present work $2 \mathrm{pm} / \mathrm{V}$ have been demonstrated in poled bismuth-zinc-borate glass planar waveguides with opportunely chosen substrates having lower electrical resistance than the guiding region. The innovative sample configuration, which provided a 4-fold improvement in the induced $\chi^{(2)}$ compared to bulk glasses [1], was suggested by a poling model that takes into account both ionic and electronic conduction. The $\chi^{(2)}$ is found to be limited by the onset of nonlinear conductivity. This work opens the way to a further enhancement of $\chi^{(2)}$ through tailoring the nonlinear conductivity via the glass composition and preparation.
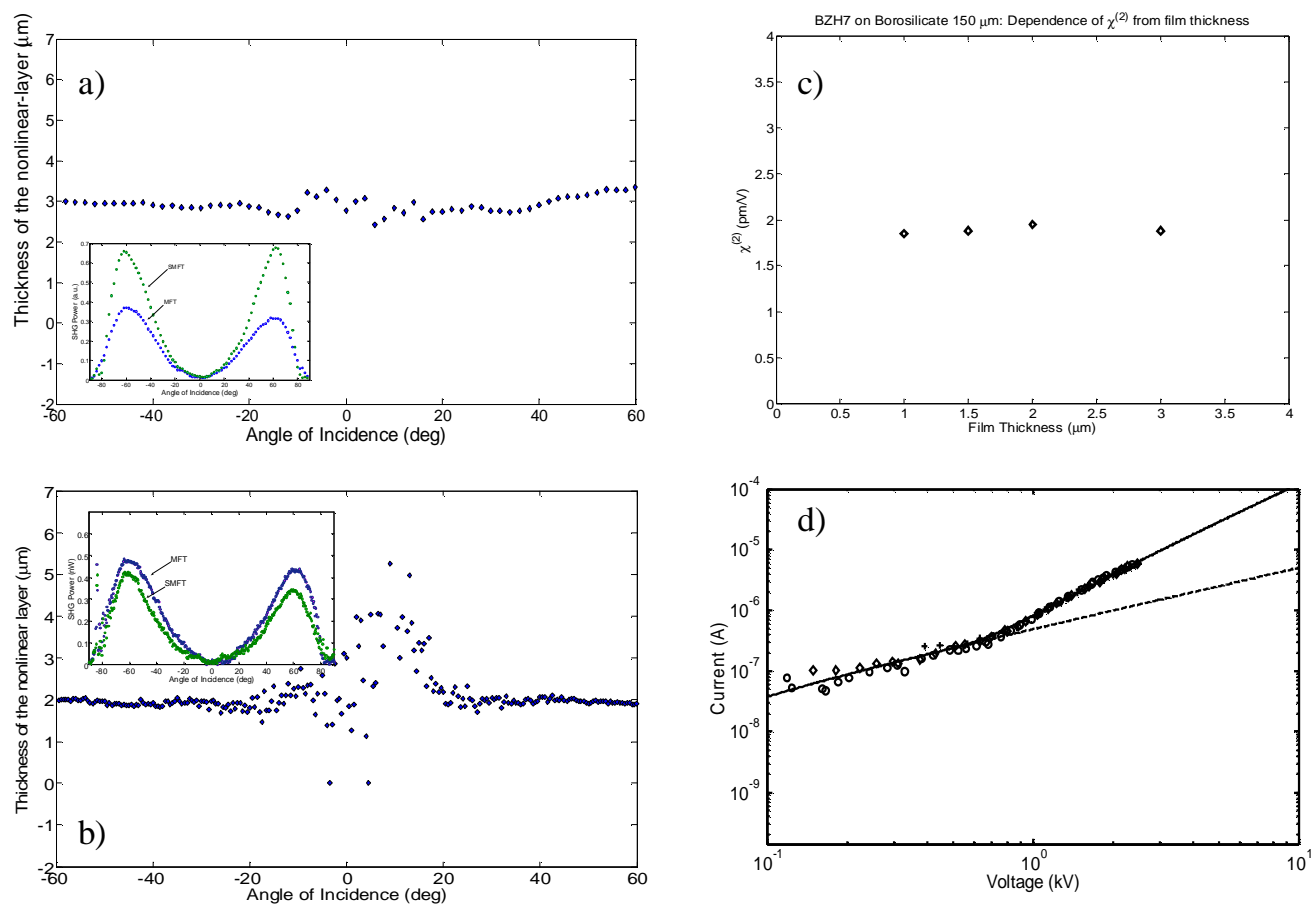

Fig. 1 Measurements of the nonlinearity and of its thickness taken by the stack Maker's fringe technique (SMFT)[2] in a) $3 \mu \mathrm{m}$ and b) $2 \mu \mathrm{m} \mathrm{BZH7}-$ film $\left(12.5 \mathrm{Bi}_{2} \mathrm{O}_{3}-43.75 \mathrm{ZnO}-43.75 \mathrm{~B}_{2} \mathrm{O}_{3}\right)$ sputtered on $150 \mu \mathrm{m}$ borosilicate substrate. The $\chi^{(2)}$ is found to extend across the whole film thickness and c) constant in value suggesting that the glass sustains a maximum electric field $\left(\mathrm{E}_{\mathrm{dc}}=\chi^{(2)} / 3 \chi^{(3)}\right)$. Current measurements during poling d) show a strong correlation between $\mathrm{E}_{\mathrm{dc}}$ and the field at which nonlinearity conductivity occurs.

A range of films of bismuth-zinc-borate glasses (supplied by NSG Ltd.) with various thickness of 1, 1.5, 2 and $3 \mu \mathrm{m}$ were grown by RF-sputtering on $150 \mu \mathrm{m}$ borosilicate substrates and poled $\left(150{ }^{\circ} \mathrm{C}, 2.5 \mathrm{kV}, 60 \mathrm{sec}\right)$. The lower electrical resistance of the substrate compared to the film provided a 4-fold enhancement of $\chi^{(2)}$ compared to bulk glass. Moreover, the induced nonlinearity extended across the whole thickness of the film without penetrating the substrate thus ensuring an optimum overlap with the guided modes of the planar waveguide (Fig. 1a, 1b). The correlation between optical and current measurements revealed that the $\chi^{(2)}$ is limited by the onset of nonlinear conductivity (Fig. 1d). Therefore tailoring of the nonlinear conductivity by optimizing glass composition and preparation can provide that further factor of 2.5 enhancements in the second-order nonlinearity that would make glass-based integrated electro-optic modulators viable.

\section{References}

[1] O.Deparis et al. "Origin and enhancement of the second-order non-linear optical susceptibility induced in bismuth borate glasses by thermal poling", Journal of Non-Crystalline Solids 2005 Vol.351(27-29) pp.2166-2177

[2] C.Corbari et al. "Practical technique for measurement of second-order nonlinearity in poled glass", Electronics Letters 2003 Vol.39(2) pp.197-198 